\title{
Dyskinesias and Treatment with Pramipexole in Patients with Parkinson's Disease
}

\author{
John C. P. Piedad ${ }^{1,2}$ and Andrea E. Cavanna ${ }^{1,3}$ \\ ${ }^{1}$ Michael Trimble Neuropsychiatry Research Group, University of Birmingham and BSMHFT, Birmingham, UK \\ ${ }^{2}$ Department of Neuropsychiatry and Barberry National Centre for Mental Health, University of Birmingham and BSMHFT, \\ 25 Vincent Drive, Birmingham B15 2FG, UK \\ ${ }^{3}$ Sobell Department of Motor Neuroscience and Movement Disorders, UCL, Institute of Neurology, London WC1N 3BG, UK
}

Correspondence should be addressed to Andrea E. Cavanna, a.cavanna@ion.ucl.ac.uk

Received 1 July 2011; Revised 30 October 2011; Accepted 14 November 2011

Academic Editor: Gilberto Fisone

Copyright ( 92012 J. C. P. Piedad and A. E. Cavanna. This is an open access article distributed under the Creative Commons Attribution License, which permits unrestricted use, distribution, and reproduction in any medium, provided the original work is properly cited.

\begin{abstract}
Dopamine agonists such as pramipexole (PPX) have first been proposed as adjunctive treatment to levodopa (L-DOPA) for patients with Parkinson's disease (PD) and then as a monotherapy alternative to alleviate dyskinesia. Treatment with PPX has overall been associated with improvement in parkinsonian symptoms. Although the majority of placebo-controlled studies demonstrated that dyskinesia was more prevalent in the PPX compared to the placebo groups, some studies did not detect any dyskinesia as a side effect of this medication. PPX was consistently associated with lower risk for developing dyskinesia compared to L-DOPA. Moreover, the presence of these symptoms in the placebo groups suggests involvement of non-PPX-related factors for developing dyskinesia. It is suggested that future research should aim at ascertaining whether cotherapy with L-DOPA, PPX dosage, and other patient characteristics are contributory factors for the development of PPX-related dyskinesia in patients with PD.
\end{abstract}

\section{Dyskinesia in Parkinson's Disease}

Parkinson's disease (PD) is a neurodegenerative disease characterised by motor (particularly tremor, rigidity, and bradykinesia) as well as cognitive and behavioural symptoms. The pathophysiology of PD has been related to the degeneration of nigrostriatal dopaminergic pathways [1], and this has allowed the treatment for PD to be targeted towards modulating dopamine (DA) neurotransmission (Figure 1).

Levodopa (L-DOPA) has long been the mainstay of PD treatment although over time patients on L-DOPA develop motor complications including dyskinesias, which are associated with the timing of drug administration. Dyskinesias are involuntary muscular contractions and include choreic, dystonic, myoclonic, and tic movements [2]. After 5 and 10 years of L-DOPA therapy, $91 \%$ and all of the participants in a longitudinal cohort $(N=99)$, respectively, experienced dyskinesias [3]. Another study also identified that cumulative L-DOPA dosage was significantly associated with the development of dyskinesia [4].
Given that dyskinesias has been consistently shown to negatively affect patients' quality of life $[5,6]$, there is considerable debate on how to forestall its onset, including initial treatment with another class of drugs: the dopamine receptor agonists (DAAs) $[7,8]$. Pramipexole (PPX) belongs to this drug class and is selective for the $\mathrm{D}_{2}$-like receptor subfamily, particularly the $\mathrm{D}_{3}$ compared to the $\mathrm{D}_{2}$ and $\mathrm{D}_{4}$ subtypes [9]. Following the observation by Hauser et al. [4] that treatment with PPX was significantly associated with later onset of dyskinesia, we carried out a systematic literature review to examine the effects of PPX therapy on dyskinetic events in patients with PD.

\section{Literature Search Methodology}

This paper systematically reviews the existing evidence on the development of dyskinesia during PPX therapy for PD. We performed a literature search across the databases Medline, EMBASE and PsycInfo via the NHS Evidence tool (http://www.library.nhs.uk). We used the search terms 


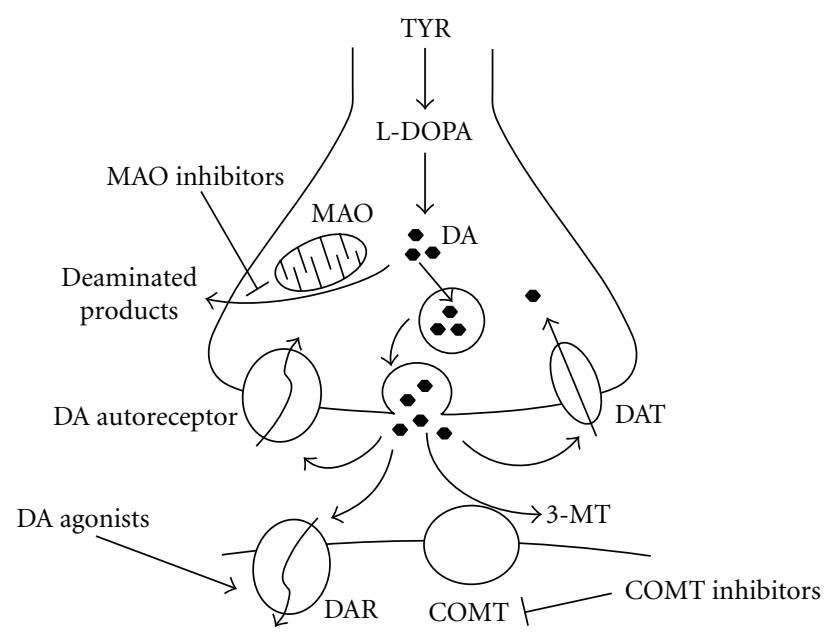

Figure 1: Treatment of Parkinson's disease: anti-Parkinson's medications modulate key stages of dopaminergic neurotransmission. Abbreviations: TYR: tyrosine, L-DOPA: L-3, 4-dihydroxyphenylalanine, DA: dopamine, MAO: monoamine oxidase, DAT: DA reuptake transporter, COMT: catechol-O-methyltransferase, 3-MT: 3-methoxytyramine, DAR: DA receptor. Anti-Parkinson's drugs are highlighted in bold. Pointed arrows indicate stimulatory, and closed arrows indicate inhibitory activity.

"Parkinson*," “dyskinesi*," and "pramipexole". The Cochrane Library was also searched for randomised and double-blind human trials of PPX in patients with PD. We limited our search to papers published in English language.

\section{Pramipexole-Placebo Comparisons}

The majority of the studies on PPX included in this review were comparisons with placebo only (Table 1). Six out of ten of these studies found that dyskinesia in PPX-treated patients was prevalent, at a higher rate than in the placebo group. The incidence of dyskinesia was $7.0-61.3 \%$ and $3.0-40.8 \%$ in the PPX and placebo groups, respectively [10-15]. The differences in rates of dyskinesia were between 4.9 and 20.5\%. Two of these studies had follow-up data. An extension to the Lieberman et al. [10] protocol by up to 50 months was carried out, in which both PPX and placebo groups were retitrated onto open-label PPX [16]. Out of the sample $(N=$ $306), 61.1 \%$ reported dyskinesias, but there were no related discontinuations. Furthermore, UPDRS IV scores remained below the baseline values, indicating some improvements in these symptoms. Möller et al. [13] reported an open-label extension to their study of up to 57 months follow-up. Out of their cohort, 34.4\% $(N=262)$ developed dyskinesias. This led to study discontinuation in $2.3 \%$ of participants. Two studies reported the incidence of dyskinesia to be higher with the placebo than the PPX groups: $0.6 \%$ versus $0.0 \%$ [17] and $6.1 \%$ versus $5.6 \%$ [18]. However, it should be noted that the strength of such evidence is weak. One study [17] was specifically designed to assess the antiparkinsonian properties in early PD, rather than the potential dyskinetic effects of pramipexole. The other one [18] found 2 dyskinetic patients in a group of 33 patients treated with placebo and 2 dyskinetic patients in a group of 36 patients treated with PPX. Of note, a further study failed to identify participants who experienced dyskinesia with PPX treatment or placebo $[19,20]$.
In terms of neurological scales as a measure of dyskinesia, Lieberman et al. [10] reported that \% change in UPDRS IV scores was significantly $(P<.0001)$ higher in the PPX group compared to the placebo group. Changes in the PDS were not statistically significant. These exact patterns in UPDRS IV and PDS scores were reproduced by Pinter et al. [11] and Möller et al. [13]. In these studies, however, the incidence of dyskinesia was higher with PPX treatment than placebo. Wermuth et al. [18] did not find significant changes in UPDRS IV or PDS scores. In a cross-over design with PPX and placebo, as well as L-DOPA infusion before and after the switch-over, Brodsky et al. [32] found that PPX treatment increased PDS scores to statistically significant levels $(P=$ .05). Furthermore, L-DOPA infusion also increased peak dyskinesia scores.

\section{Studies with Active Comparators}

4.1. Pramipexole Only. There have been two studies comparing different dosages and other two looking at different preparations of PPX. One of the earliest investigations on PPX was carried out by the Parkinson Study Group [21], which compared four different dosages of the drug $(1.5,3.0$, 4.5 , and $6.0 \mathrm{mg}$ ) against placebo. Another study by the same group compared different low-dose schedules of PPX [21]. Both did not detect any incidence of dyskinesia. Likewise, comparisons of immediate release (IR) and extended release (ER) PPX preparations failed to detect any incidence of dyskinesia symptoms in either treatment or placebo group $[26,27]$.

4.2. Pramipexole versus Other Dopamine Agonists. Other DAAs have been compared with PPX. Two studies reported a comparison with bromocriptine (BRC), showing that dyskinesias were found more often with DAAs compared with placebo. In terms of UPDRS IV and PDS scores, there were no significant changes in one trial [29], whereas, in 


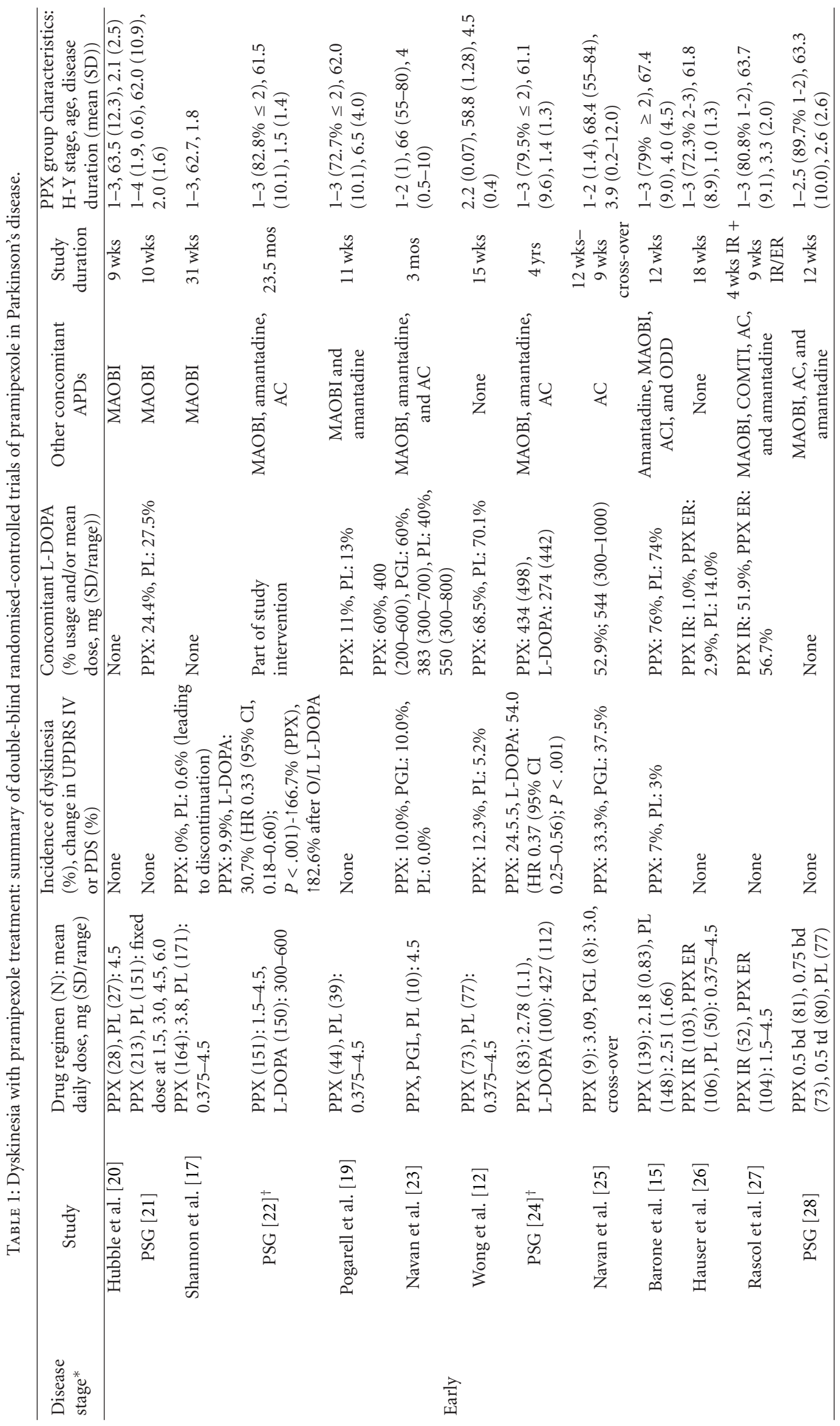




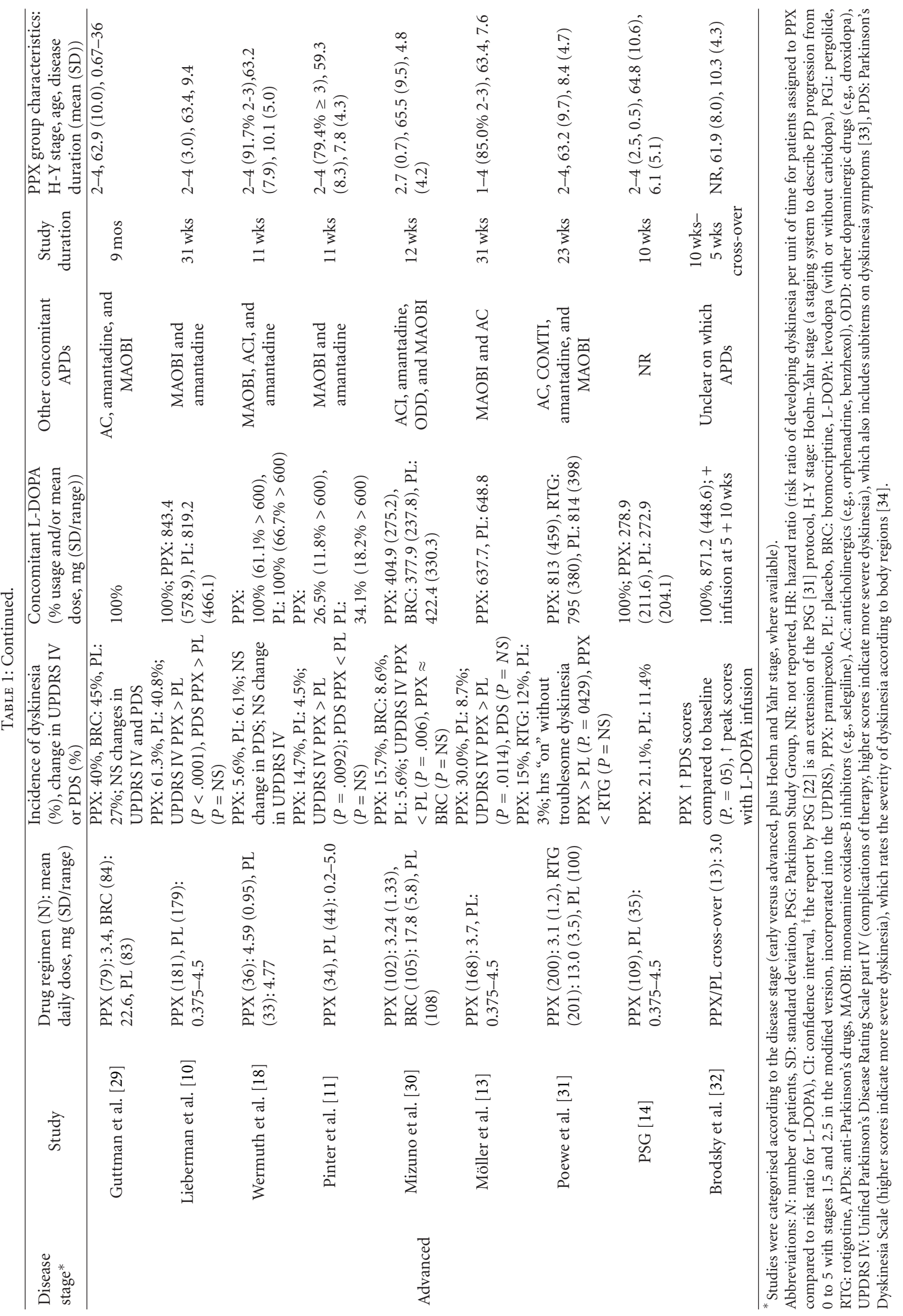


the other, \% UPDRS IV score changes were significantly lower in the PPX compared to the placebo group [30]. When comparing PPX and BRC, Guttman et al. [29] found that the incidence of dyskinesia between these treatment groups was approximately similar (5\% difference). On the other hand, dyskinesia was found to be nearly twice as prevalent with PPX treatment compared to BRC [30].

Two studies compared pergolide (PGL) with PPX. One of these studies found that dyskinesia was equally prevalent with PPX and PGL $(N=1)$ but was not reported in the placebo group [23]. Another trial by the same group with a cross-over design found an overall lower incidence of dyskinesia with PPX treatment than PGL: $33.3 \%$ versus $37.5 \%$ [25]. Transdermally administered rotigotine (RTG) was also compared with PPX [31]. The placebo group had less dyskinesia than the active treatment groups, with PPX having slightly higher incidence than RTG (3\% versus $15 \%$ versus $12 \%$ ). Furthermore, this group reported that participants in the PPX group had significantly more time in the day "on" without troublesome dyskinesias compared to placebo $(P=.0429)$, whereas the difference between PPX and RTG was not significant.

4.3. Pramipexole versus L-DOPA. Only one cohort was involved with a trial comparing PPX and L-DOPA. The first report was the two-year completion of initial treatment with PPX and L-DOPA, with open-label L-DOPA for emerging disability [22]. There was a significantly lower incidence of dyskinesia in the PPX compared to the L-DOPA group: $9.9 \%$ versus $30.7 \%$, hazard ratio (HR) 0.33 (confidence interval (CI) $0.18-0.60), P<.001$. Some of the participants who completed the two-year trial were also enrolled for a further two-year follow-up study, with the randomised and blinded protocol maintained [24]. This showed a similar pattern of the incidence of dyskinesia, which was significantly lower in the PPX $(N=83)$ compared to the L-DOPA $(N=100)$ group: $24.5 \%$ versus $54.0 \%$, HR 0.37 (95\% CI $0.25-0.56), P<.001$. None of the PPX cohort withdrew from follow-up due to dyskinesia, whereas dyskinesia-related discontinuation was found in $2.0 \%$ of the L-DOPA group. Some of the patients in the 2-and 4-year trials were also recruited to the open-label extension study with mean follow-up of 6.0 (SD 0.2) years, the majority of whom were in H-Y stages 2 [35]. Both the initial PPX $(N=108)$ and L-DOPA $(N=114)$ groups showed overall reductions in the incidence of dyskinesia: $20.4 \%$ and $36.8 \%$, respectively. Despite changes in the incidence of dyskinesia in the treatment groups compared to the previous reports of this cohort, L-DOPA treatment was associated with higher events. Furthermore, disability associated with dyskinesia in the PPX group was comparably lower than that in the L-DOPA group, which was at trend-level significance $(P=.06)$.

\section{Are There Indicators for the Development of Dyskinesia with PPX Treatment?}

The majority of placebo-controlled studies demonstrated that dyskinesias can develop during PPX treatment. The inci-
TABle 2: Pharmacokinetic profiles of selected anti-Parkinson's drugs.

\begin{tabular}{lc}
\hline APD & Half-life (hrs) \\
\hline PPX & $8-12$ \\
L-DOPA/carbidopa & $1-1.5$ \\
RTG & $5-7$ \\
PGL & $7-16$ \\
BRC & $12-15$ \\
\hline
\end{tabular}

Abbreviations: APD: anti-Parkinson's drug, PPX: pramipexole, L-DOPA: levodopa, RTG: rotigotine, PGL: pergolide, BRC: bromocriptine.

dence of these events in the placebo groups ranged between 0 and $40.8 \%$, and the differences compared to the PPX group ranged between 4.0 and $21.3 \%$. Two studies demonstrated slightly more dyskinesia in the placebo compared to the PPX group $[17,18]$. These results clearly indicate that there are non-PPX-related factors contributing to the development of dyskinesias in PD.

Treatment of L-DOPA has been proposed as an important factor for the development of dyskinesia in PD [4]. From the two trials and the long-term follow-up of their cohort $[22,24,35]$, the PSG consistently demonstrated a higher incidence of dyskinesia associated with L-DOPA therapy. Furthermore, Brodsky et al. [32] showed that PPX treatment increased L-DOPA-related dyskinesias and increased the severity and duration of dyskinesia. They hypothesised that the observed effects in their study are beyond potential additive effects, given that DAAs rarely cause dyskinesia. The 2-hour infusion of therapeutic dose L-DOPA $(1.0 \mathrm{mg} / \mathrm{kg} / \mathrm{hr})$ also produced more dyskinesia compared to subtherapeutic doses $(0.5 \mathrm{mg} / \mathrm{kg} / \mathrm{hr})$, regardless of PPX treatment. These findings suggest that L-DOPA on its own can have some effect on dyskinesia events during PPX therapy. Although a study distinguished between L-DOPA and non-L-DOPAtreated participants, the report about adverse event-related withdrawals (including dyskinesia) in the L-DOPA group was not supplemented by information about what proportions were affected [12]. The effects of L-DOPA in other studies are also less clear because L-DOPA usage is reported for the whole sample and there is no differentiation of which patients were dyskinetic.

The literature also allows limited exploration from clinical studies whether continuous dopaminergic stimulation (CDS) is a protective factor for dyskinesia [36]. CDS is a proposed strategy to prevent fluctuations in DA transmission and therefore the development of dyskinesia [37]. Studies with PPX and other DAAs as active comparators show similar incidence of dyskinesia. In all studies with placebo comparisons, DAAs have consistently been associated with more dyskinesia events, suggesting the involvement of dopaminergic activation. In terms of the pharmacokinetic profile of PPX (Table 2), its longer half-life compared to L-DOPA makes it difficult to explain the higher incidence of dyskinesias with this treatment. In fact, the shorter half-life of L-DOPA compared to other antiparkinsonian medications (such as dopamine D2 receptor agonists) is regarded as a factor contributing to its pulsatile action and 
ultimately to dyskinesia [38]. Moreover, studies on the ER preparation of PPX, which produces a continuous release of active ingredient over a twenty-four-hour period [39], did not find high rates of dyskinesia. Finally, there were no differences whether the DAA was ergoline (BRC, PGL) or non-ergoline-based (PPX, RTG). It has therefore been suggested that previous exposure to L-DOPA (i.e., priming) can lead to increased susceptibility to develop dyskinesias after exposure to drugs which would not otherwise have had this effect. Specifically, pulsatile activation of type D2 dopamine receptors is reported to be the principal factor in the triggering of dyskinesias and may well be involved in the priming phenomenon [40].

Dose-ranging studies of PPX allow some degree of examination of the hypothesis that PPX-related dyskinesia may be dosage dependent. One study examining PPX at doses of $1.5,3.0,4.5$, and $6.0 \mathrm{mg}$ did not report any incidence of dyskinesia in either treatment or placebo groups [21]. Another study of different schedules of low-dose PPX ( $0.5 \mathrm{bd}, 0.75 \mathrm{bd}$, and $0.5 \mathrm{mg}$ td) also did not report dyskinetic events in the participants [28]. These findings suggest that dyskinesia may not be PPX dose dependent. Exploration of this hypothesis with other studies is difficult because PPX dosages are reported as a mean or range of values for the whole sample. Therefore, it is not possible to determine what doses were administered to patients with dyskinesia.

Due to insufficient details in published reports, it has also been difficult to explore whether concomitant APD usage or patient characteristics (such as illness stage, age, and duration of disease) are associated with dyskinesia. APDs were either kept constant at baseline dosages or used as addon therapy for emerging disability and the majority of studies allowed concomitant usage. Furthermore, patient characteristics were reported as mean or range values without differentiating which patients exhibited dyskinesia. However, it is interesting to note that patients with dyskinesias were generally older (early sixties and above). The patients in PPXonly studies in which there was no incidence of dyskinesia were newly diagnosed with PD.

In terms of methodological issues, the duration of the treatment phase (i.e., titration and maintenance) has overall been adequate to allow sufficient time in detecting or establishing a timeframe for the onset of dyskinesia. The sample size has also generally been statistically viable to allow detection of clinically relevant findings, including dyskinetic adverse events, although there have been some studies with small sample sizes. Most studies also maintained some equivalency with patient characteristics in their treatment groups by carrying out block randomisations and statistical tests before and after treatment to investigate related differences in outcome measures.

More problematic issues, however, may confound conclusions about the effects of PPX on dyskinesia. In particular, some studies only reported adverse events including dyskinesia that occurred above threshold incidence, for example, $\geq 2 \%, \geq 5 \%$, or $10 \%$. Thus, PPX-related dyskinesias may be underreported and contribute to the lower incidence in certain studies. Indeed, the studies which did not detect dyskinesia in their participants had thresholds of reporting these events at $\geq 5 \%$ [26-28] and $10 \%$ [19]. There have also been studies in which patients already experiencing motor fluctuations including dyskinesia were enrolled for PPX treatment. These studies examined whether PPX is a suitable adjuvant medication for L-DOPA-related dyskinesia. However, from these studies it is difficult to establish whether PPX can contribute to the development of dyskinesias since it was not described whether the incidence of dyskinesia is treatment-emergent and relevant neurological scales were not used to measure effects on these symptoms. The reporting of dyskinesia in follow-up studies also does not allow the differentiation between patients with treatmentemergent events and those who continued to experience such symptoms. Additionally, the combination of ratings of dyskinesia and neurological scales to measure effects of PPX further confound conclusions without differentiating which patients had dyskinetic events. For example, the incidence of dyskinesia may be high in the PPX group even if the scales show significant improvement associated with PPX treatment $[10,11,13]$.

\section{Conclusions}

We systematically reviewed the existing evidence on the use of PPX in PD with focus on the development of dyskinesia. Treatment with PPX has overall been associated with significantly better improvement in motor and daily function compared to placebo. The majority of placebo-controlled studies demonstrated that dyskinesias can develop during PPX treatment, as these symptoms were generally (except for two studies) more prevalent compared to the placebo groups. Therefore, the evidence in support of lower incidence of dyskinesia in combination with PPX is far from convincing. However, in six studies, no dyskinesia events were reported. Four of these six studies were PPX comparisons (i.e., doseranging or immediate versus extended release preparation). Active comparator studies showed that the incidence of dyskinesia events was quite similar relative to other DAAs. An L-DOPA comparison study in one cohort with longterm follow-up consistently showed that PPX treatment was associated with lower risk for developing dyskinesia. These symptoms have also been reported in the placebo groups (albeit at generally lower rates), suggesting the potential involvement of non-PPX-related factors in the development of dyskinesia. It is still to be established whether L-DOPA treatment, PPX dosage, and other patient characteristics such as age or disease stage can play a role as contributory factors. Elucidation of such factors is likely to optimise the efficacy of anti-Parkinson's treatment and its compliance.

\section{References}

[1] J. B. Schulz, "Update on the pathogenesis of Parkinson's disease," Journal of Neurology, vol. 255, no. 5, supplement, pp. 3-7, 2008.

[2] Parkinson Study Group, "A randomised controlled trial comparing pramipexole with levodopa in early Parkinson's disease: design and methods of the CALM-PD Study," Clinical Neuropharmacology, vol. 23, no. 1, pp. 34-44, 2000. 
[3] A. Schrag, Y. Ben-Shlomo, R. Brown, C. D. Marsden, and N. Quinn, "Young-onset Parkinson's disease revisited - Clinical features, natural history, and mortality," Movement Disorders, vol. 13, no. 6, pp. 885-894, 1998.

[4] R. A. Hauser, M. P. McDermott, and S. Messing, "Factors associated with the development of motor fluctuations and dyskinesias in Parkinson disease," Archives of Neurology, vol. 63, no. 12, pp. 1756-1760, 2006.

[5] C. Marras, A. Lang, M. Krahn, G. Tomlinson, and G. Naglie, "Quality of life in early Parkinson's disease: impact of dyskinesia and motor fluctuations," Movement Disorders, vol. 19, no. 1, pp. 22-28, 2004.

[6] S. Chapuis, L. Ouchchane, O. Metz, L. Gerbaud, and F. Durif, "Impact of the motor complications of Parkinson's disease on the quality of life," Movement Disorders, vol. 20, no. 2, pp. 224230,2005 .

[7] J. L. Montastruc, O. Rascol, and J. M. Senard, "Treatment of Parkinson's disease should begin with a dopamine agonist," Movement Disorders, vol. 14, no. 5, pp. 725-730, 1999.

[8] W. J. Weiner, "The initial treatment of Parkinson's disease should begin with levodopa," Movement Disorders, vol. 14, no. 5, pp. 716-724, 1999.

[9] R. Constantinescu, "Update on the use of pramipexole in the treatment of Parkinson's disease," Neuropsychiatric Disease and Treatment, vol. 4, no. 2, pp. 337-352, 2008.

[10] A. Lieberman, A. Ranhosky, and D. Korts, "Clinical evaluation of pramipexole in advanced Parkinson's disease: results of a double-blind, placebo-controlled, parallel-group study," Neurology, vol. 49, no. 1, pp. 162-168, 1997.

[11] M. M. Pinter, O. Pogarell, and W. H. Oertel, "Efficacy, safety, and tolerance of the non-ergoline dopamine agonist pramipexole in the treatment of advanced Parkinson's disease: a double blind, placebo controlled, randomised, multicentre study," Journal of Neurology Neurosurgery and Psychiatry, vol. 66, no. 4, pp. 436-441, 1999.

[12] K. S. Wong, C. S. Lu, D. E. Shan, C. C. Yang, T. H. Tsoi, and V. Mok, "Efficacy, safety, and tolerability of pramipexole in untreated and levodopa-treated patients with Parkinson's disease," Journal of the Neurological Sciences, vol. 216, no. 1, pp. 81-87, 2003.

[13] J. C. Möller, W. H. Oertel, J. Köster, G. Pezzoli, and L. Provinciali, "Long-term efficacy and safety of pramipexole in advanced Parkinson's disease: results from a European multicenter trial," Movement Disorders, vol. 20, no. 5, pp. 602 610, 2005.

[14] C. Tanner, C. Comella, C. Kamp et al., "Pramipexole in levodopa-treated Parkinson disease patients of African, Asian, and Hispanic heritage," Clinical Neuropharmacology, vol. 30, no. 2, pp. 72-85, 2007.

[15] P. Bxarone, W. Poewe, S. Albrecht et al., "Pramipexole for the treatment of depressive symptoms in patients with Parkinson's disease: a randomised, double-blind, placebo-controlled trial," The Lancet Neurology, vol. 9, no. 6, pp. 573-580, 2010.

[16] W. J. Weiner, S. A. Factor, J. Jankovic et al., "The long-term safety and efficacy of pramipexole in advanced Parkinson's disease," Parkinsonism and Related Disorders, vol. 7, no. 2, pp. 115-120, 2001.

[17] K. M. Shannon, J. P. Bennett, and J. H. Friedman, "Efficacy of pramipexole, a novel dopamine agonist, as monotherapy in mild to moderate Parkinson's disease," Neurology, vol. 49, no. 3, pp. 724-728, 1997.

[18] L. Wermuth and The Danish Pramipexole Study Group, "A double-blind, placebo-controlled, randomised, multi-centre study of pramipexole in advanced Parkinson's disease," European Journal of Neurology, vol. 5, pp. 235-242, 1998.

[19] O. Pogarell, T. Gasser, J. J. van Hilten et al., "Pramipexole in patients with Parkinson's disease and marked drug resistant tremor: a randomised, double blind, placebo controlled multicentre study," Journal of Neurology Neurosurgery and Psychiatry, vol. 72, no. 6, pp. 713-720, 2002.

[20] J. P. Hubble, W. C. Koller, N. R. Cutler et al., "Pramipexole in patients with early Parkinson's disease," Clinical Neuropharmacology, vol. 18, no. 4, pp. 338-347, 1995.

[21] K. Kieburtz, "Safety and efficacy of pramipexole in early Parkinson disease: a randomized dose-ranging study," Journal of the American Medical Association, vol. 278, no. 2, pp. 125130, 1997.

[22] R. Holloway, I. Shoulson, K. Kieburtz et al., "Pramipexole vs Levodopa as initial treatment for Parkinson disease: a randomized controlled trial," Journal of the American Medical Association, vol. 284, no. 15, pp. 1931-1938, 2000.

[23] P. Navan, L. J. Findley, J. A. R. Jeffs, R. K. B. Pearce, and P. G. Bain, "Randomized, double-blind, 3-month parallel study of the effects of pramipexole, pergolide, and placebo on Parkinsonian tremor," Movement Disorders, vol. 18, no. 11, pp. 1324-1331, 2003.

[24] Parkinson Study Group, "Pramipexole vs levodopa as initial treatment for Parkinson disease: a 4-year randomised controlled trial," Archives of Neurology, vol. 61, pp. 1044-1053, 2004.

[25] P. Navan, L. J. Findley, M. B. Undy, R. K. B. Pearce, and P. G. Bain, "A randomly assigned double-blind cross-over study examining the relative anti-Parkinsonian tremor effects of pramipexole and pergolide," European Journal of Neurology, vol. 12, no. 1, pp. 1-8, 2005.

[26] R. A. Hauser, A. H. Schapira, O. Rascol et al., "Randomized, double-blind, multicenter evaluation of pramipexole extended release once daily in early Parkinson's disease," Movement Disorders, vol. 25, no. 15, pp. 2542-2549, 2010.

[27] O. Rascol, P. Barone, R. A. Hauser et al., "Efficacy, safety, and tolerability of overnight switching from immediate- to once daily extended-release pramipexole in early Parkinson's disease," Movement Disorders, vol. 25, no. 14, pp. 2326-2332, 2010.

[28] Parkinson Study Group, "Twice-daily, low-dose pramipexole in early Parkinson's disease: a randomized, placebo-controlled trial," Movement Disorders, vol. 26, no. 1, pp. 37-44, 2011.

[29] M. Guttman, "Double-blind comparison of pramipexole and bromocriptine treatment with placebo in advanced Parkinson's disease," Neurology, vol. 49, no. 4, pp. 1060-1065, 1997.

[30] Y. Mizuno, N. Yanagisawa, S. Kuno et al., "Randomized, double-blind study of pramipexole with placebo and bromocriptine in advanced Parkinson's disease," Movement Disorders, vol. 18, no. 10, pp. 1149-1156, 2003.

[31] W. H. Poewe, O. Rascol, N. Quinn et al., "Efficacy of pramipexole and transdermal rotigotine in advanced Parkinson's disease: a double-blind, double-dummy, randomised controlled trial," The Lancet Neurology, vol. 6, no. 6, pp. 513-520, 2007.

[32] M. A. Brodsky, B. S. Park, and J. G. Nutt, "Effects of a dopamine agonist on the pharmacodynamics of levodopa in parkinson disease," Archives of Neurology, vol. 67, no. 1, pp. 27-32, 2010.

[33] S. Fahn, R. L. Elton, and UPDRS Development Committee, "Unified Parkinson's disease rating scale," in Recent Developments in Parkinson's Disease, S. Fahn, C. D. Marsden, D. B. Calne, and M. Goldstein, Eds., pp. 153-163, Macmillan, Florham Park, NJ, USA, 1987. 
[34] W. Guy, "Abnormal involuntary movement scale," in The ECDEU Assessment Manual for Psychopharmacology, US Department of Health Education and Welfare, National Institute of Health Education and Welfare, and National Institute of Mental Health, Eds., pp. 534-537, US Government Printing Office, Washington, DC, USA, 1976.

[35] R. Holloway, K. Marek, K. Biglan et al., "Long-term effect of initiating Pramipexole vs Levodopa in early Parkinson disease," Archives of Neurology, vol. 66, no. 5, pp. 563-570, 2009.

[36] P. Jenner, "Avoidance of dyskinesia: preclinical evidence for continuous dopaminergic stimulation," Neurology, vol. 62, no. 1, pp. S47-S55, 2004.

[37] P. Barone, "Clinical strategies to prevent and delay motor complications," Neurology, vol. 61, no. 6, supplement, pp. S12S16, 2003.

[38] L. A. Smith, M. J. Jackson, L. Johnston et al., "Switching from levodopa to the long-acting dopamine D2/D3 agonist piribedil reduces the expression of dyskinesia while maintaining effective motor activity in MPTP-treated primates," Clinical Neuropharmacology, vol. 29, no. 3, pp. 112-125, 2006.

[39] W. Eisenreich, B. Sommer, S. Hartter, and W. H. Jost, "Pramipexole extended release: a novel treatment option in Parkinson's disease," Parkinson's Disease, vol. 2010, Article ID 612619, 7 pages, 2010.

[40] P. Damier, L. Tremblay, J. Féger, and E. C. Hirsch, "Development of dyskinesias induced by treatment for parkinson's disease: potential role of first exposure to L-DOPA (priming)," Revue Neurologique, vol. 156, no. 3, pp. 224-235, 2000. 


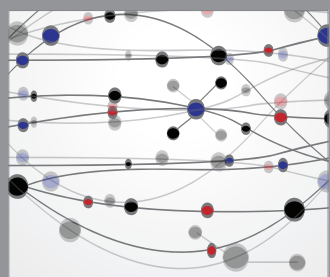

The Scientific World Journal
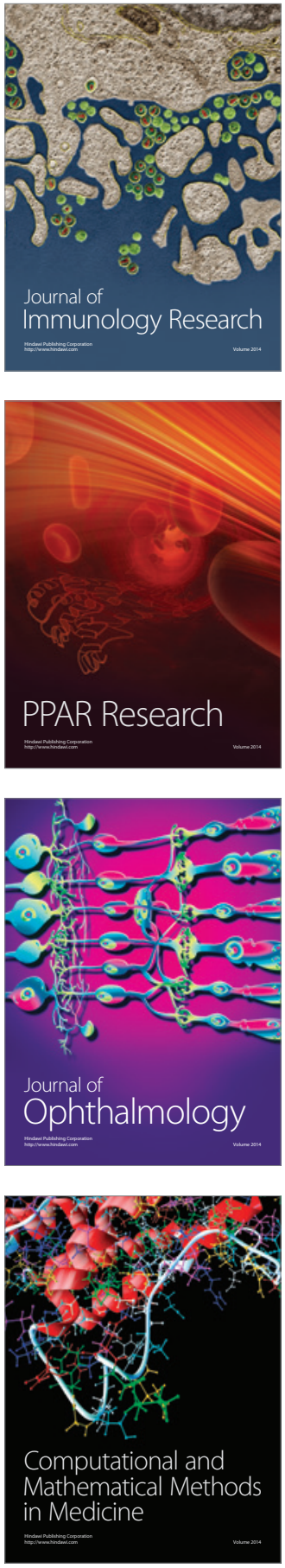

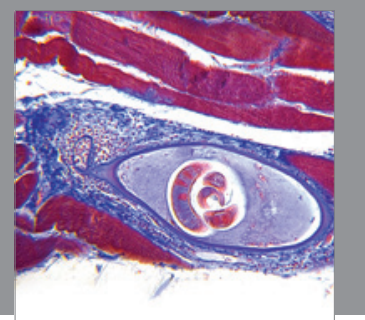

Gastroenterology

Research and Practice
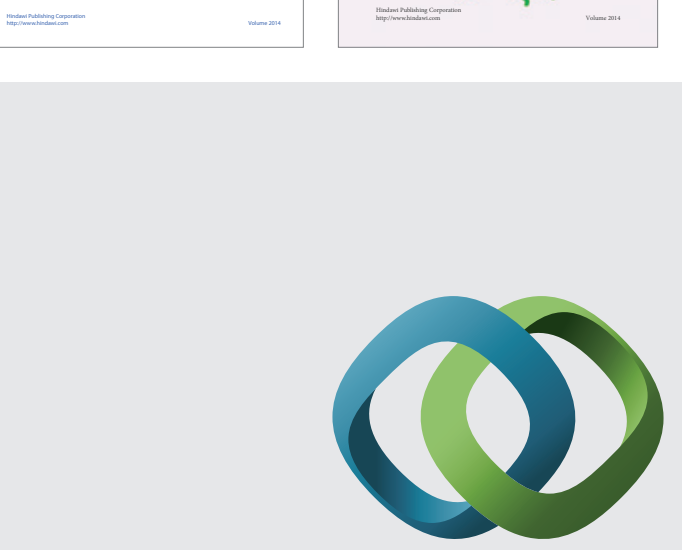

\section{Hindawi}

Submit your manuscripts at

http://www.hindawi.com
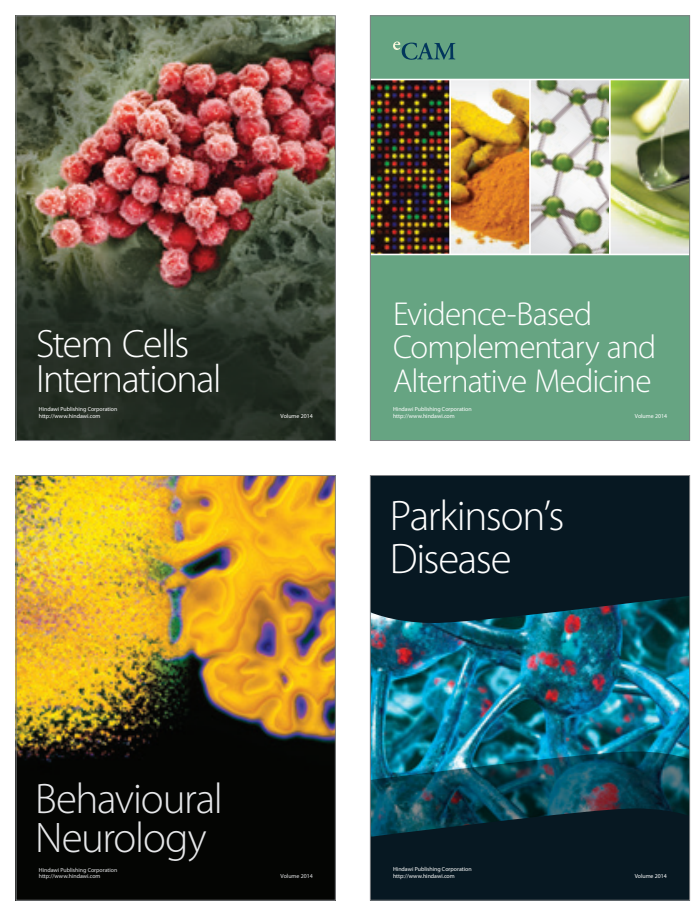

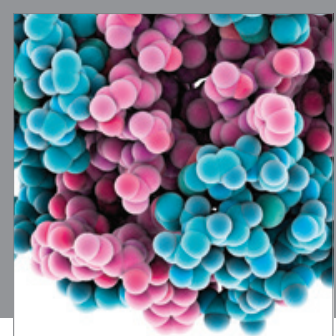

Journal of
Diabetes Research

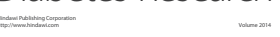

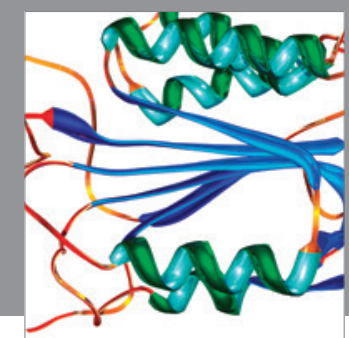

Disease Markers
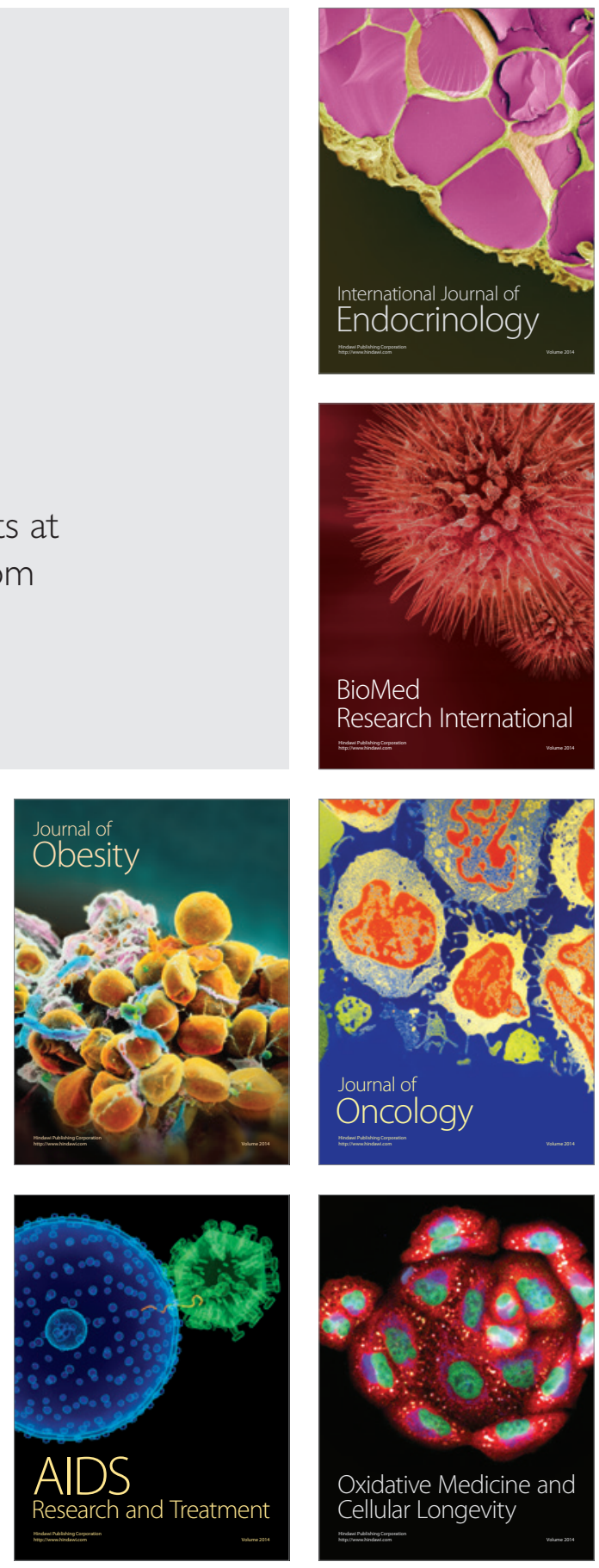